\title{
Insight into the 3D structure and substrate specificity of previously uncharacterized GNAT superfamily acetyltransferases from pathogenic bacteria.
}

Karolina A. Majorek ${ }^{1,4}$, Tomasz Osinski ${ }^{1,4}$, David T. Tran ${ }^{2}$, Alina Revilla ${ }^{2}$, Wayne F. Anderson $^{3,4}$, Wladek Minor ${ }^{1,4^{*}} \&$ Misty L. Kuhn ${ }^{2 *}$

${ }^{1}$ Department of Molecular Physiology and Biological Physics, University of Virginia, Charlottesville, VA 22908, USA

2 Department of Chemistry and Biochemistry, San Francisco State University, San Francisco, CA 94132, USA

3 Northwestern University Feinberg School of Medicine, Department of Molecular Pharmacology and Biological Chemistry, Chicago, IL 60611, USA

${ }^{4}$ Center for Structural Genomics of Infectious Diseases (CSGID)

* To whom correspondence may be addressed: Dept. of Chemistry and Biochemistry, San Francisco State University, 1600 Holloway Ave., San Francisco, CA 94132. Tel.: 415-405-2112; E-mail: mkuhn@sfsu.edu or Dept. of Molecular Physiology and Biological Physics, University of Virginia, 1340 Jefferson Park Ave., Charlottesville, VA 22908. Tel.: 434-243-6865; Fax: 434-982-1616; E-mail:

wladek@iwonka.med.virginia.edu

Keywords: acetyltransferase, Gcn5-related $N$-acetyltransferase, GNAT, amino acid acetylation, serine and threonine acetylation 


\begin{abstract}
Members of Gen5-related $N$-acetyltransferase (GNAT) superfamily catalyze the acetylation of a wide range of small molecule and protein substrates. Due to their abundance in all kingdoms of life and diversity of their functions, they are implicated in many aspects of eukaryotic and prokaryotic physiology. Although numerous GNATs have been identified thus far, many remain structurally and functionally uncharacterized. The elucidation of their structures and functions is critical for broadening our knowledge of this diverse and important superfamily. In this work, we present the structural and kinetic analyses of two previously uncharacterized bacterial acetyltransferases SACOL1063 from Staphylococcus aureus strain COL and CD1211 from Clostridium difficile strain 630. Our structures of SACOL1063 show substantial flexibility of a loop that is likely responsible for substrate recognition and binding compared to structures of other homologs. In the CoA complex structure, we found two CoA molecules bound in both the canonical AcCoA/CoA-binding site and the acceptor-substrate-binding site. Our work also provides initial clues regarding the substrate specificity of these two enzymes; however, their native function(s) remain unknown. We found both proteins act as $N$ rather than $O$-acetyltransferases and preferentially acetylate L-threonine. The combination of structural and kinetic analyses of these two previously uncharacterized GNATs provides fundamental knowledge and a framework on which future studies can be built to elucidate their native functions.
\end{abstract}




\section{Introduction}

The Gcn5-related $N$-acetyltransferase (GNAT) superfamily consists of evolutionarily related acetyltransferases from all kingdoms of life [1-3]. GNATs catalyze the transfer of an acyl group from acyl-coenzyme A (most often acetyl-coenzyme A (AcCoA)) to a primary amine or hydroxyl group, but the range of observed substrates is very wide. GNAT substrates include aminoglycoside antibiotics, polyamines, arylalkylamines, glucosamine-6-phosphate, and other small molecules [3]. GNATs are also involved in protein acetylation, including both $\mathrm{N}$-terminal $(N \alpha)$ acetylation of protein and peptide termini and $N$-epsilon $(N \varepsilon)$ acetylation of internal lysine residues [2-6]. Although the structures and functions of many GNATs have been identified, the substrate specificities and biological activities of the majority of them remain unknown. Based on the functions of GNATs characterized to date, it is clear these biological roles are very diverse and are not necessarily easy to identify.

Structural characterization of proteins often provides new insight into their function, therefore it is important to characterize new members of diverse protein superfamilies. A number of GNATs have been structurally characterized and many reveal very interesting features such as different forms of dimer formation and numerous variants of domain swapping [3]. One approach we have used to identify possible substrates for structural and functional studies of uncharacterized GNATs is to screen them using a broadsubstrate-screening assay [7]. This assay provides a mechanism to identify initial 'hits' from a variety of classes of substrates and then further characterize the enzymes both structurally and kinetically based on these 'hits.' One advantage to this assay is that it 
limits the possible classes of substrates that a GNAT uses. Even when substrates are not identified, the screen provides useful information as to what classes of substrates are not used. We previously screened ten uncharacterized GNATs from pathogenic bacteria using this assay and found that two of them preferentially acetylated L-amino acidsSACOL1063 from Staphylococcus aureus strain COL and CD1211 from Clostridium difficile strain 630. Since these proteins had neither been structurally nor functionally characterized and represent uncharacterized subgroups of the GNAT superfamily, we decided to further characterize them here. The information we gained from this set of experiments will be useful for future studies to identify the native substrates of these enzymes.

\section{MATERIALS AND METHODS}

\subsection{Materials}

All substrates were purchased from Sigma-Aldrich at the highest quality available. Acetyl coenzyme A was purchased as the tri-lithium salt.

\subsection{Cloning, expression and purification}

The SACOL1063 from Staphylococcus aureus subsp. aureus COL and CD1211 from Clostridium difficile 630 genes were cloned into the pMCSG7 pET21-derived expression vector. The vector encodes an $\mathrm{N}$-terminal $6 \times$ His-tag with a spacer followed by a tobacco etch virus (TEV) protease cleavage site. The amino acid sequence Ser-Asn-Ala remains attached to the $\mathrm{N}$-terminus of the protein after tag-cleavage with TEV protease. The fusion protein was overexpressed in E. coli BL21-RIPL (DE3) cells (Stratagene). The 
cells were grown in M9 SeMET (selenomethionine) High-Yield Growth Medium for protein that was used for crystallization and in TB for protein for kinetic characterization. Cells were induced, harvested, and the proteins were purified to near homogeneity as described previously $[7,8]$. The TEV cleaved protein was passed through a Superdex 200 column in a buffer containing $10 \mathrm{mM}$ Tris- $\mathrm{HCl} \mathrm{pH} 7.5$ and $150 \mathrm{mM} \mathrm{NaCl}$. After gel filtration, fractions containing the protein were pooled and concentrated to 18 and 9 mg/ml (SACOL1063 and CD1211, respectively).

\subsection{Site-directed mutagenesis}

Mutants of SACOL1063 and CD1211 were generated using the QuikChange site-directed mutagenesis kit (Stratagene) according to the manufacturer's instructions. The wild-type genes were used as templates and the presence of mutations was confirmed by DNA sequencing.

\subsection{Crystallization}

We used the Xtaldb [9] and LabDB [10] to track protein preparation and all crystallization experiments. The crystals of SACOL1063 were grown at $16^{\circ} \mathrm{C}$ using vapor diffusion and a sitting drop setup for the apo-form, and hanging drop for CoAcomplex. The crystallization drops were a 1:1 mixture of protein solution and the precipitant solution from the wells (0.2 M Sodium thiocyanate and 20\% PEG3350 in the case of the apo form, and 0.1 M Bis-tris propane $\mathrm{pH} 7.0$ and 2.2 M DL-Malic acid in case of the CoA-complex). The apo form crystal grew due to micro-seeding process that occurred on the fiber of unknown source that was present in the crystallization drop. For the structure of the complex with CoA, the SACOL1063 protein was incubated on ice 
with $10 \mathrm{mM}$ CoA for 1 hour prior to crystallization setup. Prior to data collection, each crystal was transferred to a solution containing a 2:1 mixture of precipitant solution and ethylene glycol for the apo structure and LV Cryo Oil (MiTeGen) for the CoA-complex. Crystals were immediately cryo-cooled in liquid nitrogen.

\subsection{Data collection, structure determination and refinement}

Data collection was performed at the 19-ID beam line of the Structural Biology Center [11] at the Advanced Photon Source (APS). Data were collected at a temperature of 100 K and processed with HKL-2000 [12]. The structures were determined by Se singlewavelength anomalous diffraction (SAD) using HKL-3000 [13] integrated with SHELEXC/D/E [14, 15], CCP4 [16] and ARP/wARP [17]. Refinement was performed using HKL-3000 coupled with REFMAC5 [18], COOT [19, 20], and selected programs from the CCP4 package [16]. The B-factors were refined using Translation/Libration/Screw (TLS) groups assigned by the TLSMD server [21]. Validation of the structures was performed using MOLPROBITY [22] and ADIT [23]. The coordinates and structures factors have been deposited in the Protein Data Bank. (PDB codes: 5JQ4, 5JPH). Statistics describing crystallographic data collection and refinement are summarized in Table 2.

\subsection{Activity screening and measurement of kinetic activity}

To determine the kinetic parameters of the SACOL1063 and CD1211 enzymes, we produced substrate saturation curves for substrates identified from the previous broadscreening assay (see section 3.8 and (19)) by varying concentration of one substrate while holding the concentration of the other substrate constant. For instance, if we were testing 
the kinetic parameters for AcCoA, we varied its concentration $(0-2 \mathrm{mM})$ while holding the acceptor substrate concentration constant at $10 \mathrm{mM}$. Alternatively, when we tested kinetic parameters for acceptor substrates, we varied their concentrations $(0-10 \mathrm{mM})$ while holding AcCoA concentration constant at $1 \mathrm{mM}$. Curves were produced in triplicate and data were fitted to a modified Hill equation as described previously [8]. In order to compare our data from before, all assays were performed as described previously $[7,8]$ in $50 \mu \mathrm{L}$ reaction mixtures containing $50 \mathrm{mM}$ Tris- $\mathrm{HCl} \mathrm{pH} 8.0$ buffer, $10 \mathrm{mM}$ acceptor substrate, and $1 \mathrm{mM} \mathrm{AcCoA}$ unless otherwise stated. Reactions were allowed to proceed for $10 \mathrm{~min}$ at $37^{\circ} \mathrm{C}$ and were initiated with $10 \mu \mathrm{l}$ of enzyme (final concentration of WT SACOL1063 and CD1211 enzymes was $0.19 \mu \mathrm{M}$ and $0.084 \mu \mathrm{M}$ ).

In order to determine the kinetic mechanism for each enzyme we produced a series of AcCoA substrate saturation curves at set concentrations of L-Thr $(2.5,5,10$, and $20 \mathrm{mM})$ in triplicate using the same reaction conditions and concentrations of enzymes as described above. The data were fitted to a set of kinetic models as described previously [24] to determine which model was most compatible with the data. To evaluate whether conserved tyrosine residues were critical for catalysis, we compared specific activities of SACOL1063 WT, SACOL1063 Y114F, CD1211 WT, and CD1211 Y133F enzymes in triplicate. Reactions contained $50 \mathrm{mM}$ Tris- $\mathrm{HCl} \mathrm{pH} 8.0$ buffer, $10 \mathrm{mM} \mathrm{L}-\mathrm{Thr}$, and $1 \mathrm{mM}$ AcCoA. The final concentration of enzyme in each reaction was $0.12 \mu \mathrm{M}, 0.11 \mu \mathrm{M}, 1.2$ $\mu \mathrm{M}$, or $1.1 \mu \mathrm{M}$, respectively. Protein from the monomer peaks for SACOL1063 and CD1211 after gel-filtration chromatography was used for all kinetic assays. Under the described assay conditions, one unit of enzyme activity is defined as one $\mu \mathrm{mol}$ of CoA produced per minute. 


\subsection{Sequence analyses}

Searches of the NCBI non-redundant (nr) database, using the sequences of SACOL1063 and CD1211 proteins as queries, were performed using PSI-BLAST [25] with a stringent expectation (e)-value threshold of 1e-30. These sequences were used for phylogenetic distribution analysis and to select sequences for the alignment. A multiple sequence alignment (MSA) of selected sequences was generated using PROMALS3D [26] and manually adjusted. In order to analyze the occurrence of particular amino acids in Ntermini of proteins in Clostridium difficile strain 630 and Staphylococcus aureus strain COL we downloaded all protein sequences for these strains from the NCBI Protein database. We removed identical sequences to obtain a non-redundant data set and calculated the incidence of each amino acid in the position following the $\mathrm{N}$-terminal Met.

\subsection{Modeling of CD1211 protein structure}

Secondary structure prediction and Fold-Recognition (FR) analysis of CD1211 were carried out via the GeneSilico Meta-Server gateway (for references to original methods see https://genesilico.pl/meta2) [27]. FR alignments to the top-scoring templates from the Protein Data Bank were compared, evaluated and ranked by PCONS [28]. Based on the FR analysis the structures of PaiA acetyltransferase from Bacillus subtilis (PDB ID: 1TIQ) and the tabtoxin resistance protein from Pseudomonas syringae (PDB ID: 1GHE) were chosen as templates for modeling the CD1211 structure. Alignments reported by FR methods were manually refined to maximize the number of aligned homologous residues and preserve the continuity of predicted secondary structure elements, and they were then used for automated comparative modeling using MODELLER [29]. Energy minimization of the model was performed using MOE (Molecular Operating Environment (47). 
software with an AMBER99 force field to a root mean square deviation gradient of 0.1 $\mathrm{kcal} / \mathrm{mol} / \AA^{2}$.

In order to assess the model accuracy, we used the meta-server MetaMQAP that obtains scores from a number of third-party model quality assessment programs and calculates a predicted deviation between the position of each residue in the computational model and its position in the real (unknown) structure [30]. Independently, global evaluation of model quality was performed with the PROQ method [31]. For crude modeling of the CD1211:AcCoA complex, the CD1211 apo protein model was superimposed with the structure $1 \mathrm{GHE}$ and the AcCoA was copied from the 1GHE structure. Energy minimization of AcCoA molecule was performed using MOE with an MMF94x force field, followed by energy minimization of the whole complex with an AMBER99 force field, and again energy minimization of the AcCoA alone with MMF94x. The resulting CD1211:AcCoA complex was considered an approximation of how CD1211 will interact with AcCoA.

\section{RESULTS}

\subsection{Identification and sequence comparison of SACOL1063 and CD1211 related} proteins

The Staphylococcus aureus (strain COL) gene SACOL1063 encodes a 144 amino acid protein classified as a member of the UPF0039 (ElaA) family in UniProtKB and the PF00583 acetyltransferase family in the Pfam database. Homologs of SACOL1063 were identified using a PSI-Blast search of the non-redundant (nr) database using a stringent expectation (e)-value threshold of $1 \mathrm{e}-30$ and the phylogenetic distribution of the 
identified proteins were analyzed (Supplementary Table 1). The data set comprised mostly sequences from Bacteria, but some sequences from Archaea and Eukaryota were also present. The Archaeal sequences included proteins that were predominantly from Halobacteriaceae, but also included some from Methanosarcina. The Eukaryotic proteins were mainly from Fungi, Euglenozoa, Amebozoa, and Oomycetes. The sequence alignment of selected proteins from organisms representing each of these groups is presented in Figure 1A.

The Clostridium difficile (strain 630) gene CD1211 encodes a 159 amino acid protein, which is also classified as a member of the PF00583 acetyltransferase family in the Pfam database. The phylogenetic distribution based on a stringent PSI-Blast search indicated that the closest homologs of CD1211 (Supplementary Table 2) are present in Bacteria and some Eukaryota, but not in Archaea. The Eukaryotic proteins are found mostly in Fungi, but our search also identified a few proteins from Chlorella, Trichoplax and one protein from Acanthamoeba. Figure 1B shows the sequence alignment of CD1211 homologs selected from organisms representing each of these taxonomic groups.

\subsection{Overall structure of SACOL1063}

In the apo-form, SACOL1063 crystallized in the space group C2 with two molecules in the asymmetric unit. SACOL1063 has a standard GNAT fold with the core $\beta$-sheet composed of seven, mainly antiparallel $\beta$-strands, and helices on both sides of the central $\beta$-sheet. When we compared the two molecules in the asymmetric unit, we observed a significant difference in the conformations of the loop between $\beta 6$ and $\beta 7$ (residues 121- 
135) (Figure 2A). Moreover, when we displayed two symmetry-related molecules we saw that this loop binds in the cavity of the neighboring protein molecule in both molecules, even though the conformation of the loop is different in each of them. The Cys 108 residues from the two molecules of the asymmetric unit are positioned next to each other and form a disulfide bond (Figure 2B). The way the loop binds to the neighboring molecule causes it to block both the AcCoA-binding site and the acceptorsubstrate-binding site (Figure 2C). However, the conformation of the loop is likely a result of crystal packing and it does not necessarily represent the exact conformation of the enzyme in solution. Since this flexible loop blocks the active site of the protein, we were unable use this crystal form for the crystallographic study of the interactions with ligands.

\subsection{Structure of SACOL1063 in complex with CoA}

We co-crystallized the SACOL1063 protein in the presence of CoA in the space group $\mathrm{P} 22_{1}$ with three protein molecules in the asymmetric unit. The conformation of the protein in all three molecules was the same and each protein molecule had two molecules of CoA bound - one in the canonical AcCoA/CoA-binding site and one in the acceptor-substratebinding site (Figure 3A). The thiol groups of the two CoA molecules are directed towards

each other with a distance of $2.9 \AA$ between the two sulphur atoms (Figure 3B). The CoA binding mode in the AcCoA/CoA-binding site is similar to previously determined GNAT structures in complex with $\mathrm{AcCoA} / \mathrm{CoA}[1,32]$. The classical structural characteristic features of GNATs are maintained with a " $\beta$-bulge" in $\beta 4$ and the splay between $\beta 4$ and $\beta 5$ strands that creates a V-shaped cleft in the $\beta$-sheet to provide a binding site for the 
pantetheine of CoA. The pantetheine makes hydrogen bonds with the main chain of $\beta 4$, mimicking an antiparallel $\beta$-strand. The conformation of CoA bound in the AcCoA/CoAbinding site, with flexion of the pantothenate moiety, is also characteristic for GNATs. The adenosine moiety of CoA is located on the surface of the protein and interacts with the protein only through water molecules. The pyrophosphate moiety of CoA is coordinated mostly by the main chain nitrogen atoms of the conserved phosphate binding loop (Arg78-Gly79-Gln80-Gly81-Met82-Gly83) between $\beta 4$ and $\alpha 3$ (the conserved motif in GNATs is $(\mathrm{Q} / \mathbf{R})-\mathrm{X}-\mathrm{X}-\mathbf{G}-\mathrm{X}-(\mathbf{G} / \mathrm{A}))$ [33, 34]. Arg84, which is not part of the GNAT motif, coordinates the pyrophosphate with its main chain nitrogen and the 3'phosphate of the ribose with its side chain.

The CoA molecule bound in the acceptor-substrate-binding site is very sharply bent (Figure 3B), and the closest distance between the pantothenate and adenine groups is 3.4 $\AA$. Binding CoA in this site displays an interaction of the pyrophosphate of CoA with Asn105 and an interaction of the ribose of CoA with Glu129. The majority of the interactions of $\mathrm{CoA}$ in the acceptor-substrate-binding site come from the pantothenate moiety with Asp35, Arg57, and Arg70 (Figure 3C). The combination of these hydrogen bonding interactions with $\mathrm{CoA}$ in the acceptor-substrate-binding site suggests that $\mathrm{CoA}$ may mimic substrate binding. If so, these residues that interact with $\mathrm{CoA}$ may be important for substrate recognition in this site.

\subsection{Comparison of SACOL1063 apo- and CoA structures}

In the apo-SACOL1063 structure, we observed substantial flexibility of the loop between 
$\beta 6$ and $\beta 7$. This loop adopts a different conformation upon binding $\mathrm{CoA}$ in the acceptorsubstrate-binding site compared the apo structure. Binding CoA in the acceptor-substrate site causes $\beta 6$ and $\beta 7$ to become extended and the loop between them shortens significantly. Additionally, the entire $\beta 6$-loop- $\beta 7$ fragment shifts much closer to $\alpha 1$ and $\alpha 2$ helicies (residues 18-35) and 'closes' the acceptor-substrate-binding site (Figure 4A). These regions of the protein are conserved in SACOL1063 homologs, which may suggest their importance in substrate recognition and binding. The conformation of the $\beta 6$-loop$\beta 7$ fragment in this 'closed' form is very similar to its conformation in structures of homologous proteins, especially the PA0115 N-acyltransferase from Pseudomonas aeruginosa (PDB ID: 1XEB) (Figure 4B). The protein structures of SACOL1063 homologs, including the PA0115 protein, an uncharacterized $N$-acetyltransferase $\mathrm{YjcF}$ from Bacillus subtilis (PDB ID: 1Q2Y), and an uncharacterized $N$-acetyltransferase from Lactobacillus plantarum (PDB ID: 3EFA), all display a similar conformation of this loop even though they do not contain ligands bound in the acceptor-substrate-binding site. A comparison of the conformation of this loop in both apo-form molecules and the CoAbound form shows that this region is highly flexible and suggests that binding substrates may induce a conformational change.

\subsection{Comparison of SACOL1063 structure to other GNATs}

A structural similarity search of the PDB using DALI [35] revealed that SACOL1063 showed the highest structural similarity to several uncharacterized GNATs. The hits with the highest scores included the structure of the uncharacterized $N$-acetyltransferase YjcF from B. subtilis (PDB ID: 1Q2Y; root mean square deviation (rmsd) value of $1.5 \AA$ and 
$38 \%$ identity between the two sequences), an uncharacterized $\mathrm{N}$-acetyltransferase from L. plantarum (PDB ID: 3EFA; rmsd of $1.6 \AA$ and $27 \%$ identity), and the PA0115 Nacyltransferase from P. aeruginosa (PDB ID: 1XEB; rmsd of $1.8 \AA$ and $29 \%$ identity). Since the GNAT fold is relatively conserved, SACOL1063 also showed some structural similarity to known proteins, including protein $N \alpha$-acetyltransferases RimI from S. enterica (PDB ID: 2CNS [34]; rmsd $2.3 \AA$ and $17 \%$ identity), RimL from S. enterica (PDB ID: 1S7N [36]; rmsd $2.9 \AA$ and 8\% identity), and RimJ from V. fischeri (PDB ID: 3IGR; rmsd $2.7 \AA$ and 9\% identity), as well as the histone acetyltransferase TGCN5 from T. thermophila (PDB ID: 1M1D; rmsd $2.7 \AA$ and $15 \%$ identity). The largest differences between the Rim structures and SACOL1063 were observed in helices $\alpha 1$ and $\alpha 2$.

\subsection{Comparative model of CD1211}

Despite significant efforts, we were unable to obtain suitable crystals for data collection of the CD1211 protein in the apo-form or in complex with AcCoA/CoA. There are no close homologs of CD1211 (e.g. with 30\% or higher sequence identity) with a known structure. Therefore, to gain insight as to the positions of residues potentially important for the activity of the CD1211 enzyme, we used bioinformatics methods to produce a comparative (homology) model of the CD1211 structure and its complex with AcCoA. Fold-recognition (FR) methods, which try to find folds of the known protein structures that are compatible with a particular sequence of interest, revealed clear similarity of CD1211 to other GNATs [27]. Based on the FR analysis, we chose structures of the PaiA acetyltransferase from B. subtilis (PDB ID: 1TIQ) [37] and the tabtoxin resistance protein from P. syringae (PDB ID: 1GHE) [38] as templates for modeling the CD1211 structure. 
The alignments for most of the protein sequence were unambiguous, but there were some differences that were most prominent in the $\alpha 2$ helix and the loop between $\alpha 1$ and $\alpha 2$ helicies between the template and CD1211protein. Since no structure of a close homolog has been determined, the accuracy of the prediction in this region may be lower than for the remainder of the protein structure because it is most variable between different GNATs. The model of the CD1211 protein was evaluated using MetaMQAP [30] and PROQ [31] and is shown in Figure 5A. MetaMQAP predicted a very high global accuracy of our model, with an rmsd to "real structure" predicted to be $\sim 2.1 \AA$ and the GDT_TS (global distance test total score) predicted to be 80.7. The PORQ program gave an LG (Levitt-Gerstein) score of 5.3, indicating a potentially "extremely good" model.

The main differences between the modeled CD1211 structure and the templates used to produce the model are in the $\alpha 2$ helix, the loop between $\alpha 1$ and $\alpha 2$ helicies, and the length of the loop between $\beta 6$ and $\beta 7$ (Figure 5A). These loops are shorter in CD1211, which could potentially allow binding of a larger substrate. The optimized alignment with the templates is shown in Figure 1B. We also prepared a model of the structure of the CD1211:AcCoA complex (see section 2.8). Based on this model, we could unambiguously determine that the conserved Tyr133 is perfectly positioned to play an important role in catalysis. We also analyzed conservation of other residues in the acceptor-substrate-binding site between the closest homologs of CD1211. The residues that are conserved in this region and could potentially play a role in catalysis or substrate recognition and binding include Glu83, Lys85, Arg86, Asp121 and the more distant Glu44 (Figure 1B, Figure 5B). Comparison of structures of SACOL1063 and CD1211 
show similarities; however, the acceptor-substrate-binding pocket is in a more "open" conformation in CD1211 compared to the more "closed" CoA bound form of SACOL1063 (Figure 6).

\subsection{Oligomeric states of SACOL1063 and CD1211}

We performed gel-filtration chromatography during purification of both SACOL1063 and CD1211 proteins and found SACOL1063 eluted as monomers, whereas the CD1211 protein eluted as predominantly monomers but a subpopulation of dimers also eluted (Supplementary Figure 1). This result indicates that the CD1211 protein may exist in multiple oligomeric states. If the apo protein structure of SACOL1063 is used as a query in the PISA server [39], dimeric or tetrameric states are predicted to be the stable oligomeric forms; however, the large surface of the interaction between the two molecules in the asymmetric unit is likely caused by contacts that are the result of crystal packing. Therefore, they are most likely not biologically relevant. When the CoA-bound form is used as a query, the server predicts the protein is monomeric. The combination of gel filtration data and structural analysis suggests that the SACOL1063 protein functions as a monomer.

\subsection{Substrate specificity and kinetic parameters of SACOL1063 and CD1211 proteins}

Since the native acetyl-acceptor substrates for SACOL1063 and CD1211 are unknown, we previously performed a broad-substrate screening assay to identify potential classes of substrates for these enzymes [7]. The assay is composed of a variety of compounds including antibiotics, polyamines, organic building blocks, all 20 standard amino acids, 
small peptides, and other metabolites. Our prior screening revealed that both enzymes had a preference for acetylating some of the 20 standard L-amino acids [7], but we did not perform further kinetic assays to determine substrate specificity or kinetic parameters since the aim was strictly screening in nature. During our prior screening, we identified L-threonine, L-tryptophan, and L-tyrosine as possible substrates for SACOL1063 and Lserine, $O$-acetyl-L-serine, L-threonine, L-methionine, L-tryptophan, L-valine, Lisoleucine, L-leucine, L-homoserine, 7-aminocephalosporanic acid, thiamine pyrophosphate, and thiamine as possible substrates for CD1211 [7]. Here, we kinetically characterized the enzymes to determine substrate specificity and kinetic parameters of both enzymes.

SACOL1063 showed a strong preference for acetylating L-Thr and displayed a one order of magnitude lower kcat and catalytic efficiency for L-Trp (Table 1). The enzyme also exhibited positive cooperativity toward AcCoA in the presence of L-Thr, whereas no cooperativity was observed toward AcCoA in the presence of L-Trp (Table 1). Additionally, we determined that L-Tyr was not a viable substrate for SACOL1063 under the described assay conditions. Given that SACOL1063 was active toward L-Thr and LTrp but not $N$-methyl-L-Thr and $N$-acetyl-L-Trp [7], we can conclude that SACOL1063 is specific for acetylating the alpha amino group of amino acids and is therefore an $\mathrm{N}$ - not $O$-acetyltransferase.

During our kinetic characterization of CD1211, we found the enzyme acetylated L-Thr, L-Ser, $O$-acetyl-L-Ser, and L-Met with comparable catalytic efficiencies (Table 1). The highest efficiencies were displayed toward L-Thr and L-Ser, and the kcat toward L-Thr was nearly 3.2-, 17.8-, and 32.5-fold higher compared to L-Ser, $O$-acetyl-L-Ser, and L- 
Met, respectively (Table 1). The CD1211 enzyme was not active under the described assay conditions toward the remainder of the compounds identified as possible substrates from the broad-substrate screening assay. Unlike the SACOL1063 enzyme that exhibited positive cooperativity toward AcCoA in the presence of only L-Thr, CD1211 displayed positive cooperativity toward AcCoA in the presence of all four substrates-L-Thr, L-Ser, $O$-acetyl-L-Ser, and L-Met (Table 1). Effectively no cooperativity was observed for either SACOL1063 or CD1211 toward L-Thr (Table 1). This indicates SACOL1063 and CD1211 only exhibit positive cooperativity toward AcCoA, not both substrates of the bisubstrate reaction. This type of asymmetric cooperativity, where the enzyme only displays cooperativity toward one of the two substrates of a bisubstrate reaction, has been previously observed in some pyruvate kinases [40, 41].

The CD1211 enzyme was active toward L-Thr, L-Ser, and L-Met, but not $N$-methyl-LThr, $N$-methyl-L-Ser, and $N$-acetyl-L-Met from the previous screening assay [7]; therefore, the enzyme acts as an $N$-acetyltransferase by acetylating the alpha amino group of the amino acids. The compound $O$-acetyl-L-Ser has a modified side chain hydroxyl group and free alpha amino and alpha carboxyl groups available for additional acetylation. Since CD1211 displays a relatively similar catalytic efficiency toward this compound compared to L-Ser, the enzyme activity is not significantly affected when the side chain of L-Ser is modified. This may be important when identifying the native substrate of this enzyme for two main reasons: 1) if CD1211 acts as an N-terminal protein acetyltransferase the protein substrate's N-terminal residue could be either unmodified or post-translationally modified at this position, and 2) if the enzyme acetylates a nonprotein substrate the modification of the side chain of $O$-acetyl-L-Ser may provide clues 
regarding substituents that are acceptable in this position.

\subsection{Catalytic residues and kinetic mechanism}

The mechanism of acetyl transfer that is considered to be most common in GNATs is one in which the acetyl group is transferred directly from the AcCoA donor to a primary amine of the acceptor. In this mechanism the ternary complex of the enzyme, which contains acceptor-substrate and AcCoA, is formed followed by nucleophilic attack of the primary amine on the carbonyl carbon of AcCoA. Both SACOL1063 and CD1211 have a Tyr residue that is conserved and suitably positioned in the structure for a role in catalysis. The corresponding Tyr in this position in other GNATs has been shown to be important $[8,34]$, and it likely functions as a general acid by donating a proton to the thiolate anion of CoA formed after the transfer of the acetyl group. To determine if Tyr114 (SACOL1063) and Tyr133 (CD1211) were important for catalysis, we replaced these residues with phenylalanine and measured their specific activity compared to the wildtype proteins in the presence of $1 \mathrm{mM} \mathrm{AcCoA}$ and $10 \mathrm{mM}$ L-Thr. The specific activity of the SACOL1063 Y114F mutant decreased 176-fold compared to the WT protein $(8.20$ $\mathrm{U} / \mathrm{mg}$ compared to $0.0466 \mathrm{U} / \mathrm{mg}$ ). The specific activity of the CD1211 Y133F mutant decreased 8080-fold compared to the WT protein $(19.3 \mathrm{U} / \mathrm{mg}$ compared to 0.00239 $\mathrm{U} / \mathrm{mg}$ ). These results indicate that the conserved tyrosine residues in both SACOL1063 and CD1211 proteins are indeed critical for catalysis.

To determine whether the SACOL1063 and CD1211 enzymes used the common direct transfer kinetic mechanism or an alternative mechanism that involves an acyl-enzyme 
intermediate, we tested the fitting of a set of kinetic models using a previously described procedure [24]. We used data obtained from AcCoA substrate saturation curves in the presence of different concentrations of L-Thr (Figure 7). The most compatible model for SACOL1063 was a bireactant random steady-state model (Model VIII, [24]) and a bireactant random steady-state 2 model (Model IX, [24]) for CD1211 (Figure 7, Supplementary Table 3, Supplementary Figure 1), which indicates that both enzymes prefer a direct transfer kinetic mechanism. The minor differences between these two models indicate that both enzymes share a common core kinetic mechanism to acetylate L-Thr. For both enzymes the rate of product formation $\left(\mathrm{k}_{\mathrm{p}}\right)$ is much greater than the rate constants of the formation of intermediates, and as a result the enzymes prefer a steadystate rather than rapid equilibrium kinetic mechanism. The binding order of L-Thr or AcCoA is random for both enzymes, and either path will produce product. However, it appears that the path of binding AcCoA prior to L-Thr may be preferred because less buildup of intermediate will occur compared to binding L-Thr first.

\section{DISCUSSION}

Although many GNATs have been identified in all kingdoms of life, the biological activities of many of them remain unknown and are very diverse. To provide a framework for downstream studies of GNATs from pathogenic bacteria, our laboratories seek to determine the 3D structures of uncharacterized bacterial GNATs and study their kinetic properties. Our contribution toward addressing the need for increased functional and structural characterization of bacterial GNATs is presented in this work where we identified and characterized two prokaryotic $N$-acetyltransferases from Gram-positive 
pathogenic bacteria - SACOL1063 from S. aureus and CD1211 C. difficile. These proteins represent two different and previously uncharacterized subgroups within the large superfamily of GNATs.

Using X-ray crystallography, we determined the structure of SACOL1063 in the apoform and in complex with CoA. In the CoA complex, we observed two molecules of $\mathrm{CoA}$ - one bound in the canonical $\mathrm{AcCoA} / \mathrm{CoA}$ binding site, and one bound in the acceptor-substrate-binding site. A significant conformational change of this loop upon binding $\mathrm{CoA}$ in the acceptor-substrate-binding site occurs, and the manner in which the protein interacts with $\mathrm{CoA}$ in this site suggests how the enzyme may interact with its substrate. The loop that 'closes' the acceptor-substrate-binding site upon binding CoA in this site was observed to be substantially more flexible in the apo structure and its conformation has not been observed in structures of homologous proteins. Both SACOL1063 and CD1211 proteins were extremely difficult to crystallize. The flexibility of the aforementioned loop in SACOL1063 may be a culprit in the inability to form suitable crystals. In the case of the SACOL1063 apo structure the crystal grew due to micro seeding, while for the CoA complex the loop was stabilized by the bound CoA. We were unable to obtain suitable crystals of CD1211 for data collection despite numerous attempts, therefore we used bioinformatics methods to generate a comparative (homology) model of CD1211 to gain insight into its three dimensional structure.

We previously screened the SACOL1063 and CD1211 proteins to identify potential substrates and found that they both preferentially acetylate amino acids [7]; however, a 
thorough kinetic characterization with these substrates was not performed until now. The broad-substrate screen used for identification of potential substrates provides a starting point for further kinetic characterization and narrows the possible classes of substrates for analysis to a more manageable number. Our results showed SACOL1063 has a very narrow substrate specificity compared to CD1211, which could acetylate several amino acids (L-Thr, L-Ser, $O$-acetyl-L-Ser, and L-Met). Although both SACOL1063 and CD1211 showed higher activity with L-Thr, they exhibit different kinetic characteristics. We analyzed the phylogenetic distribution of the proteins that showed high sequence similarity to our enzymes and found when SACOL1063 was used as a query we identified its homolog from Clostridium difficile 630 that was about $30 \%$ identical (GI: 126699123). However, when CD1211 was used as a query we did not identify any similar protein in Staphylococcus aureus. These two distinct enzymes from different GNAT subgroups are likely involved in very specific roles within their respective organisms.

Since these enzymes both $\mathrm{N}$-acetylate the alpha amino group of amino acids, it is tempting to speculate that they may be involved in N-terminal protein acetylation $(\mathrm{N \alpha}$ acetylation), but they may also acetylate other substrates like antimicrobial peptides, lipopeptides, other metabolites, or catalyze the acylation of lipoamino acids. Antimicrobial peptides are host defense molecules naturally found in all living organisms, but are also common as synthetic alternatives to other antimicrobial agents like classical antibiotics. Antimicrobial peptides undergo numerous post-translational modifications, including $\mathrm{N}$-acetylation, that change their physicochemical properties [42]. N $\alpha$ - 
acetylation occurs when an acetyl group is transferred from AcCoA to the $\mathrm{N}$-terminal $(\mathrm{N} \alpha)$ amine of a protein substrate. It is one of the earliest modifications that can occur during protein synthesis and is considered an irreversible modification that is either on the N-terminal methionine or the consecutive residue after removal of the initial methionine $[2,4,6,43]$. Although $\mathrm{N}$-terminal protein acetylation is relatively well characterized in eukaryotes, little is known about it in bacteria [2, 4, 6, 43]. Recently, $N \alpha$ acetylation in bacteria has been described as being more abundant than previously recognized and a number of substrate proteins have been identified [5]; however, knowledge regarding which enzymes catalyze these reactions remain obscure.

We wanted to know if L-Thr Na-acetylation could be possible in $S$. aureus and $C$. difficile, therefore, we analyzed the occurrence of each amino acid in the position following the N-terminal Met in the $S$. aureus strain COL and the C. difficile strain 630 (Figure 8). The incidence of Thr in the position after the initiator Met residue in all proteins of S. aureus strain COL is more than $11 \%$, which, if SACOL1063 is a protein $N \alpha$-acetyltransferase, may reflect the tighter substrate specificity of SACOL1063 for LThr. Distribution of other amino acids in the C. difficile strain 630 is generally similar to that of S. aureus strain COL; however, Thr is only present in the position following the N-terminal Met in just over 2\% of proteins from C. difficile strain 630.

\section{CONCLUSIONS}

The physiological substrates for both SACOL1063 and CD1211 enzymes remain to be determined, but our work provides a foundation that will be beneficial for interpreting 
structural and kinetic details of how these enzymes may function. Further characterization of these proteins in the context of their in vivo environment may reveal the true substrates for these enzymes and increase our understanding of their function(s) in bacteria or within a host.

\section{ACKNOWLEDGEMENTS}

We thank Dr. Sergii Pshenychnyi at the Recombinant Protein Production Core at Northwestern University for protein expression and purification services. This project was funded in whole or in part with Federal funds from the National Institute of Allergy and Infectious Diseases, National Institutes of Health (NIH), Department of Health and Human Services, under Contracts No. HHSN272200700058C and HHSN272201200026C (CSGID). Additional funding for this project includes San Francisco State University Startup Funds (to MLK). Some results shown in this report are derived from work performed at the Structural Biology Center Sector 19 at the Advanced Photon Source. The Advanced Photon Source, an Office of Science User Facility, is operated for the U.S. Department of Energy (DOE) Office of Science by Argonne National Laboratory. Argonne is operated by UChicago Argonne, LLC, for the U.S. DOE Office of Biological and Environmental Research under contract DE-AC02-06CH11357.

\section{FIGURE LEGENDS}

Figure 1. Sequence alignments of selected proteins from organisms representing SACOL1063 (A) and CD1211 (B) homologs. Sequences are denoted by a five-letter abbreviation for genus and species followed by GI number. The positions showing at least $80 \%$ identity/similarity are background-colored. Secondary structure elements were determined using STRIDE [44] and are shown above the alignment with $\alpha$-helices represented by tubes, $\beta$-strands by arrows, and loops by continuous lines.

Figure 2. Structure of SACOL1063 in the apo form. (A) Superposition of each molecule in the asymmetric unit, (B) SACOL1063 (bright colors) with the symmetryrelated molecules (pale colors). Cys108 residues that form disulfide bridges are shown as red sticks. (C) SACOL1063 apo structure containing both molecules in the asymmetric 
unit (gray and red) with CoA copied from the superimposed structure of the SACOL1063 CoA complex. CoA is shown as sticks, with cyan carbon atoms of the CoA bound in the canonical AcCoA-binding site and yellow carbon atoms of CoA bound in the canonical acceptor-substrate-binding site. The loop between $\beta 6$ and $\beta 7$ of one molecule in the asymmetric unit (red) blocks both the AcCoA-binding and the acceptor-substrate-binding site of the neighboring molecule (gray).

Figure 3. Structure of SACOL1063 in complex with CoA. (A) Two CoA molecules were bound - one in the canonical AcCoA/CoA-binding site (cyan), and one in the acceptor-substrate-binding site (yellow). 2Fo-Fc electron density map of the bound CoA molecules is presented $(\sigma=1.0)$. (B) The CoA molecule bound in the acceptor-substratebinding site (yellow) is very sharply bent. The distance of $2.9 \AA$ between the two sulphur atoms of the two CoA molecules, and the closest distance $(3.4 \AA)$ between the pantothenate and adenine groups of the CoA molecule bound in the acceptor-substratebinding site are indicated. (C) Residues involved in binding of $\mathrm{CoA}$ in the acceptorsubstrate-binding site.

Figure 4. Superposition of SACOL1063 structures. (A) Superposition of SACOL1063 structures in the apo form (chains A (blue) and B (green)) and in the CoA-bound form (gray). Conformational change of the loop between $\beta 6$ and $\beta 7$ is observed upon CoA binding. (B) Superposition of SACOL1063 structure in the CoA-bound form (gray) with the PA0115 N-acyltransferase from Pseudomonas aeruginosa (PDB ID: 1XEB) (purple). A similar conformation of the loop between $\beta 6$ and $\beta 7$ is visible.

Figure 5. Homology model of CD1211. (A) The model is colored according to the predicted accuracy from blue (highly confident) to red (predicted low accuracy). AcCoA (orange sticks) was copied from the tabotoxin resistance protein structure (PDB ID: $1 \mathrm{GHE}$ ) and the entire model was subjected to energy minimization (see section 2.8). The frames indicate the region showing the most significant differences between CD1211 and the corresponding region of the homologs - the tabotoxin resistance protein structure (PDB ID: 1GHE) (pink) and the PaiA acetyltransferase template structure (PDB ID:1TIQ) (green). (B) Position of conserved residues of CD1211 that are directed toward the acceptor-substrate-binding site and may play a role in substrate recognition and/or binding. Catalytic Tyr133 is also indicated.

Figure 6. Comparison of homology model of CD1211 with structure of SACOL1063. (A) SACOL1063 in CoA bound form (gray) and CD1211 homology model (blue), (B) overlay of SACOL1063 in CoA bound form (gray), SACOL1063 in apo-form (magenta), and CD1211 homology model (blue).

Figure 7. Series of AcCoA curves at varying concentrations of L-threonine for both SACOL1063 and CD1211. AcCoA curves are shown in the presence of 2.5, 5, 10, or 20 $\mathrm{mM} \mathrm{L-Thr}$ as filled circles, open triangles, filled triangles, and open upside down triangles, respectively. The fitting to the most compatible models (ones with the lowest AICc values; Supplementary Table 3) is shown: The models that were most compatible with the data are shown below the graphs: SACOL1063 to the bireactant steady-state model and CD1211 data to the bireactant steady-state 2 model. Equations for each model are shown in [24]. Kinetic parameters obtained from the model for SACOL1063 include 
$\mathrm{K}_{\mathrm{a}}(0.77 \mu \mathrm{M}), \mathrm{K}_{\mathrm{b}}(53 \mu \mathrm{M}), \mathrm{c}(3.0), \mathrm{k}_{\text {off_a }}\left(0.72 \mathrm{~s}^{-1}\right), \mathrm{k}_{\text {off_b }}\left(1.1 \mathrm{~s}^{-1}\right)$, and $\mathrm{k}_{\mathrm{p}}\left(66 \mathrm{~s}^{-1}\right)$. Calculated rate constants for the top path include $\mathrm{k}_{\text {on_a }}\left(0.93 \mathrm{~s}^{-1} \mu \mathrm{M}^{-1}\right)$ and $\mathrm{k}_{\text {on_b }}(0.0070 \mathrm{~s}$ $\left.{ }^{1} \mu \mathrm{M}^{-1}\right)$ and rate constants for the bottom path are $\mathrm{k}_{\text {on_a }}\left(0.31 \mathrm{~s}^{-1} \mu \mathrm{M}^{-1}\right)$ and $\mathrm{k}_{\text {on_b }}\left(0.021 \mathrm{~s}^{-}\right.$ $\left.{ }^{1} \mu \mathrm{M}^{-1}\right)$. Kinetic parameters obtained from the model for CD1211 include $\mathrm{K}_{\mathrm{a}}(0.064 \mu \mathrm{M})$, $\mathrm{K}_{\mathrm{b}}(8.5 \mu \mathrm{M}), \mathrm{c}(5.0), \mathrm{k}_{\text {off_al }}\left(0.25 \mathrm{~s}^{-1}\right), \mathrm{k}_{\text {off_a2 }}\left(1.1 \mathrm{~s}^{-1}\right), \mathrm{k}_{\text {off_b1 }}\left(0.96 \mathrm{~s}^{-1}\right), \mathrm{k}_{\text {off_b2 }}\left(0.78 \mathrm{~s}^{-1}\right)$, and $\mathrm{k}_{\mathrm{p}}\left(15 \mathrm{~s}^{-1}\right)$. Calculated rate constants include $\mathrm{k}_{\text {on_al }}\left(3.9 \mathrm{~s}^{-1} \mu \mathrm{M}^{-1}\right), \mathrm{k}_{\text {on_a2 }}\left(3.3 \mathrm{~s}^{-1} \mu \mathrm{M}^{-1}\right)$, $\mathrm{k}_{\text {on_b1 }}\left(0.11 \mathrm{~s}^{-1} \mu \mathrm{M}^{-1}\right)$, and $\mathrm{k}_{\text {on_b2 }}\left(0.018 \mathrm{~s}^{-1} \mu \mathrm{M}^{-1}\right)$.

Figure 8. The incidence of each amino acid in the position following the $\mathbf{N}$-terminal Met. Staphylococcus aureus strain COL (blue) and Clostridium difficile strain 630 (red).

\section{TABLES}

Table 1. Kinetic parameters of SACOL1063 and CD1211 enzymes.

Table 2. Crystallographic data and refinement statistics for SACOL1063 structures. Values in parenthesis refer to the highest resolution shell. AU=asymmetric unit. 


\section{REFERENCES}

[1] M.W. Vetting, S.d.C. LP, M. Yu, S.S. Hegde, S. Magnet, S.L. Roderick, J.S. Blanchard, Structure and functions of the GNAT superfamily of acetyltransferases, Arch Biochem Biophys, 433 (2005) 212-226.

[2] J. Soppa, Protein acetylation in archaea, bacteria, and eukaryotes, Archaea, 2010 (2010).

[3] A.I. Salah Ud-Din, A. Tikhomirova, A. Roujeinikova, Structure and Functional Diversity of GCN5-Related N-Acetyltransferases (GNAT), International journal of molecular sciences, 17 (2016).

[4] J. Hollebeke, P. Van Damme, K. Gevaert, N-terminal acetylation and other functions of Nalpha-acetyltransferases, Biol Chem, 393 (2012) 291-298.

[5] T. Ouidir, F. Jarnier, P. Cosette, T. Jouenne, J. Hardouin, Characterization of Nterminal protein modifications in Pseudomonas aeruginosa PA14, Journal of proteomics, 114 (2015) 214-225.

[6] J.D. Jones, C.D. O'Connor, Protein acetylation in prokaryotes, Proteomics, 11 (2011) 3012-3022.

[7] M.L. Kuhn, K.A. Majorek, W. Minor, W.F. Anderson, Broad-substrate screen as a tool to identify substrates for bacterial Gcn5-related N-acetyltransferases with unknown substrate specificity, Protein Sci, 22 (2013) 222-230.

[8] K.A. Majorek, M.L. Kuhn, M. Chruszcz, W.F. Anderson, W. Minor, Structural, functional and inhibition studies of a GNAT superfamily protein PA4794: a new Cterminal lysine protein acetyltransferase from Pseudomonas aeruginosa, J Biol Chem, (2013).

[9] M.D. Zimmerman, M. Chruszcz, K.D. Koclega, Z. Otwinowski, W. Minor, The Xtaldb system for project salvaging in high-throughput crystallization, Acta Crystallogr. Sect. A, 61 (2005) c178-c179.

[10] M.D. Zimmerman, M. Grabowski, M.J. Domagalski, E.M. Maclean, M. Chruszcz, W. Minor, Data management in the modern structural biology and biomedical research environment, Methods in molecular biology, 1140 (2014) 1-25.

[11] G. Rosenbaum, R.W. Alkire, G. Evans, F.J. Rotella, K. Lazarski, R.G. Zhang, S.L. Ginell, N. Duke, I. Naday, J. Lazarz, M.J. Molitsky, L. Keefe, J. Gonczy, L. Rock, R. Sanishvili, M.A. Walsh, E. Westbrook, A. Joachimiak, The Structural Biology Center 19ID undulator beamline: facility specifications and protein crystallographic results, J Synchrotron Radiat, 13 (2006) 30-45.

[12] Z. Otwinowski, Minor W., Processing of X-ray diffraction data collected in oscillation mode., in: Methods in enzymology: Macromolecular crystallography, part A, vol. 276, Academic Press, New York, 1997, pp. 307-326.

[13] W. Minor, M. Cymborowski, Z. Otwinowski, M. Chruszcz, HKL-3000: the integration of data reduction and structure solution--from diffraction images to an initial model in minutes, Acta Crystallogr D Biol Crystallogr, 62 (2006) 859-866.

[14] G.M. Sheldrick, A short history of SHELX, Acta Crystallogr A, 64 (2008) 112-122.

[15] G.M. Sheldrick, Experimental phasing with SHELXC/D/E: combining chain tracing with density modification, Acta Crystallogr D Biol Crystallogr, 66 (2010) 479-485.

[16] N. Collaborative Computional Project, The CCP4 suite: programs for protein crystallography., Acta Crystallogr, Sect D: Biol. Crystallogr., 50 (1994) 760-763. 
[17] A. Perrakis, R. Morris, V.S. Lamzin, Automated protein model building combined with iterative structure refinement, Nat Struct Biol, 6 (1999) 458-463.

[18] G.N. Murshudov, P. Skubak, A.A. Lebedev, N.S. Pannu, R.A. Steiner, R.A. Nicholls, M.D. Winn, F. Long, A.A. Vagin, REFMAC5 for the refinement of macromolecular crystal structures, Acta Crystallogr D Biol Crystallogr, 67 (2011) 355-367.

[19] P. Emsley, K. Cowtan, Coot: model-building tools for molecular graphics, Acta Crystallogr D Biol Crystallogr, 60 (2004) 2126-2132.

[20] P. Emsley, B. Lohkamp, W.G. Scott, K. Cowtan, Features and development of Coot, Acta Crystallogr D Biol Crystallogr, 66 (2010) 486-501.

[21] J. Painter, E.A. Merritt, TLSMD web server for the generation of multi-group TLS models, Journal of Applied Crystallography, 39 (2006) 109-111.

[22] I.W. Davis, A. Leaver-Fay, V.B. Chen, J.N. Block, G.J. Kapral, X. Wang, L.W. Murray, W.B. Arendall, 3rd, J. Snoeyink, J.S. Richardson, D.C. Richardson, MolProbity: all-atom contacts and structure validation for proteins and nucleic acids, Nucleic Acids Res, 35 (2007) W375-383.

[23] H. Yang, V. Guranovic, S. Dutta, Z. Feng, H.M. Berman, J.D. Westbrook, Automated and accurate deposition of structures solved by X-ray diffraction to the Protein Data Bank, Acta Crystallographica Section D, 60 (2004) 1833-1839.

[24] E.V. Filippova, M.L. Kuhn, J. Osipiuk, O. Kiryukhina, A. Joachimiak, M.A. Ballicora, W.F. Anderson, A novel polyamine allosteric site of SpeG from Vibrio cholerae is revealed by its dodecameric structure, J Mol Biol, 427 (2015) 1316-1334.

[25] S.F. Altschul, T.L. Madden, A.A. Schaffer, J. Zhang, Z. Zhang, W. Miller, D.J. Lipman, Gapped BLAST and PSI-BLAST: a new generation of protein database search programs, Nucleic Acids Res, 25 (1997) 3389-3402.

[26] J. Pei, B.H. Kim, N.V. Grishin, PROMALS3D: a tool for multiple protein sequence and structure alignments, Nucleic Acids Res, 36 (2008) 2295-2300.

[27] M.A. Kurowski, J.M. Bujnicki, GeneSilico protein structure prediction meta-server, Nucleic Acids Res, 31 (2003) 3305-3307.

[28] J. Lundstrom, L. Rychlewski, J. Bujnicki, A. Elofsson, Pcons: a neural-networkbased consensus predictor that improves fold recognition, Protein Sci, 10 (2001) 23542362.

[29] A. Sali, T.L. Blundell, Comparative protein modelling by satisfaction of spatial restraints, J Mol Biol, 234 (1993) 779-815.

[30] M. Pawlowski, M.J. Gajda, R. Matlak, J.M. Bujnicki, MetaMQAP: a meta-server for the quality assessment of protein models, BMC Bioinformatics, 9 (2008) 403.

[31] B. Wallner, A. Elofsson, Can correct protein models be identified?, Protein Sci, 12 (2003) 1073-1086.

[32] F. Dyda, D.C. Klein, A.B. Hickman, GCN5-related N-acetyltransferases: a structural overview, Annu Rev Biophys Biomol Struct, 29 (2000) 81-103.

[33] A.F. Neuwald, D. Landsman, GCN5-related histone N-acetyltransferases belong to a diverse superfamily that includes the yeast SPT10 protein, Trends Biochem Sci, 22 (1997) $154-155$.

[34] M.W. Vetting, D.C. Bareich, M. Yu, J.S. Blanchard, Crystal structure of RimI from Salmonella typhimurium LT2, the GNAT responsible for N(alpha)-acetylation of ribosomal protein S18, Protein Sci, 17 (2008) 1781-1790.

[35] L. Holm, P. Rosenstrom, Dali server: conservation mapping in 3D, Nucleic Acids 
Res, 38 (2010) W545-549.

[36] M.W. Vetting, L.P. de Carvalho, S.L. Roderick, J.S. Blanchard, A novel dimeric structure of the RimL Nalpha-acetyltransferase from Salmonella typhimurium, J Biol Chem, 280 (2005) 22108-22114.

[37] F. Forouhar, I.S. Lee, J. Vujcic, S. Vujcic, J. Shen, S.M. Vorobiev, R. Xiao, T.B. Acton, G.T. Montelione, C.W. Porter, L. Tong, Structural and functional evidence for Bacillus subtilis PaiA as a novel N1-spermidine/spermine acetyltransferase, J Biol Chem, 280 (2005) 40328-40336.

[38] H. He, Y. Ding, M. Bartlam, F. Sun, Y. Le, X. Qin, H. Tang, R. Zhang, A. Joachimiak, J. Liu, N. Zhao, Z. Rao, Crystal structure of tabtoxin resistance protein complexed with acetyl coenzyme A reveals the mechanism for beta-lactam acetylation, $\mathrm{J}$ Mol Biol, 325 (2003) 1019-1030.

[39] E. Krissinel, K. Henrick, Inference of macromolecular assemblies from crystalline state, J Mol Biol, 372 (2007) 774-797.

[40] M.E. Munoz, E. Ponce, Pyruvate kinase: current status of regulatory and functional properties, Comparative biochemistry and physiology. Part B, Biochemistry \& molecular biology, 135 (2003) 197-218.

[41] I. Faustova, J. Järv, Kinetic analysis of cooperativity of phosphorylated L-type pyruvate kinase., Proc. Estonian Acad. Sci. Chem., 55 (2006) 179-189.

[42] G. Wang, Post-translational Modifications of Natural Antimicrobial Peptides and Strategies for Peptide Engineering, Current biotechnology, 1 (2012) 72-79.

[43] R.A. Bradshaw, W.W. Brickey, K.W. Walker, N-terminal processing: the methionine aminopeptidase and $\mathrm{N}$ alpha-acetyl transferase families, Trends Biochem Sci, 23 (1998) 263-267.

[44] M. Heinig, D. Frishman, STRIDE: a web server for secondary structure assignment from known atomic coordinates of proteins, Nucleic Acids Res, 32 (2004) W500-502. 
A

SACOL1063 APO SACOL1063 COA SACOL 1063

Cldif_515100160 Vicho_518665585 Accal 488044997 Thnea_221572272 Eninv_471194307 Trcru_71650313 Scpom_19113711 Phsoj_348665945 Sccom_302696909 Hasal_496824602 NaTai_493880268 Memaz_505225876 Lapla_206582109 Psaer_56554506

SACOL1063 APO SACOL1063 COA SACOL 1063

Cldif_515100160 Vicho_518665585 Accal_488044997 Thnea_221572272 Eninv_471194307 Trcru_71650313 Scpom_19113711 Phsoj_348665945 Sccom_302696909 Hasal_496824602 NaTai_493880268 Memaz_505225876 Lapla_206582109 Psaer_56554506

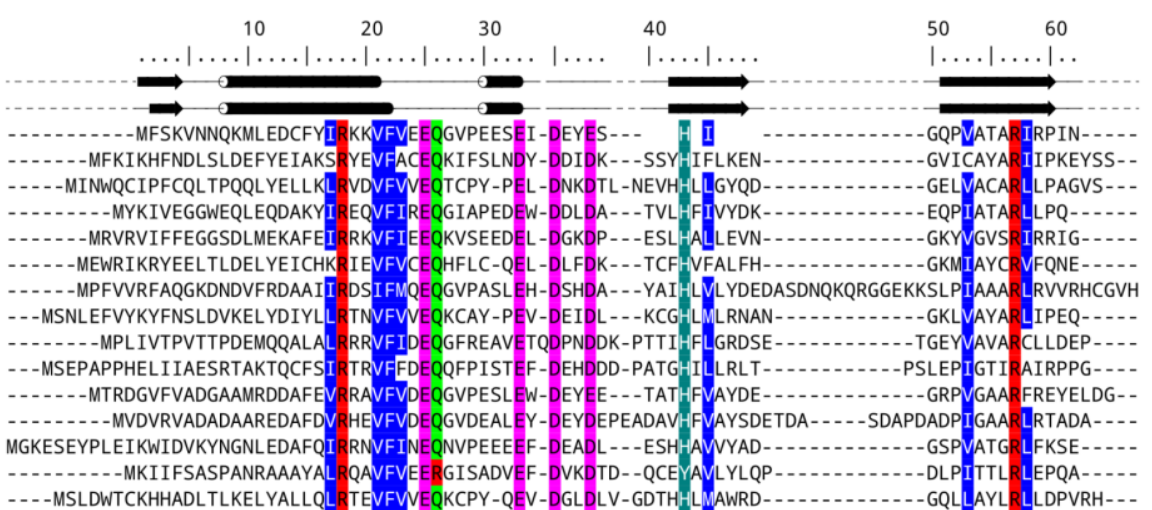

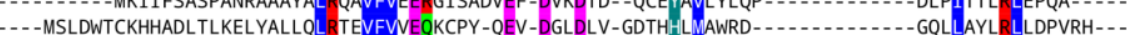

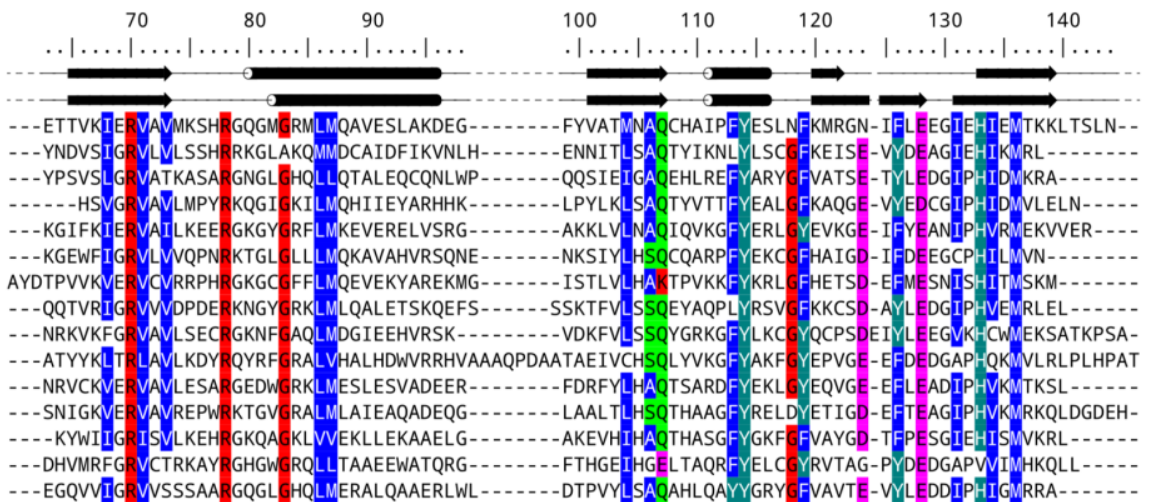

B

CD1211

CD1211

Labre_227190651

Funec_386907918

Bucen_105896428

Spjap_292677471 Deapa_518418615

Scpom_19111935

Asory_169767104

Trvir_358385012

Chvar_307102315

Tradh_196008275

1GHE_A

$1 T$ T A

CD1211

Labre_227190651

Funec_386907918

Bucen_105896428

Spjap_292677471

Deapa_518418615 -

Scpom_19111935 - -

Trar_307102315 HTPTLGEGDAVAGVPLAEVCEM

Tradh_196008275

GHE_A

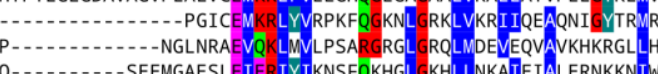

20

30
$40 \quad 50$

50
$1 \ldots$

60

CFQDFNNELKTLPGKYKK - ....

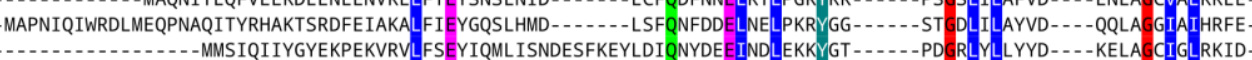
- - MLALFPVISRRPMIAIRAARYPDDAAAVEAIFREYVASPTVS- - - LAFQDYEPEIAALPGKYAA- - - - PRGQLLLAWRG - - ERVVGCAAFREIDAVNATTLSYCRHDFRRSNHAMVVIRHAIFPRDTASVLGIWREFIANSPVN - - - LDYQNNDAEFANLPGKYAA - - - PKGCVLLADRE- - GEIEGCVAMRQVT

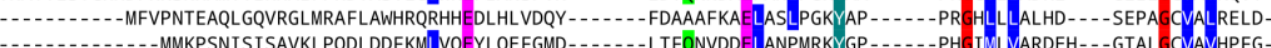

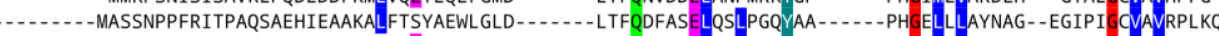
- MATLPTATSSVLVRQATSPSDLAAVVECFKEYTAWLDED- - - LTHQNYTAELNGLPGKYAA - - - PTGALLLAVDAT - TDAVLGCVAVRPISL

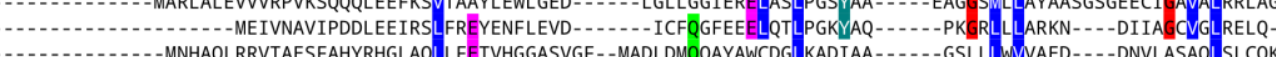

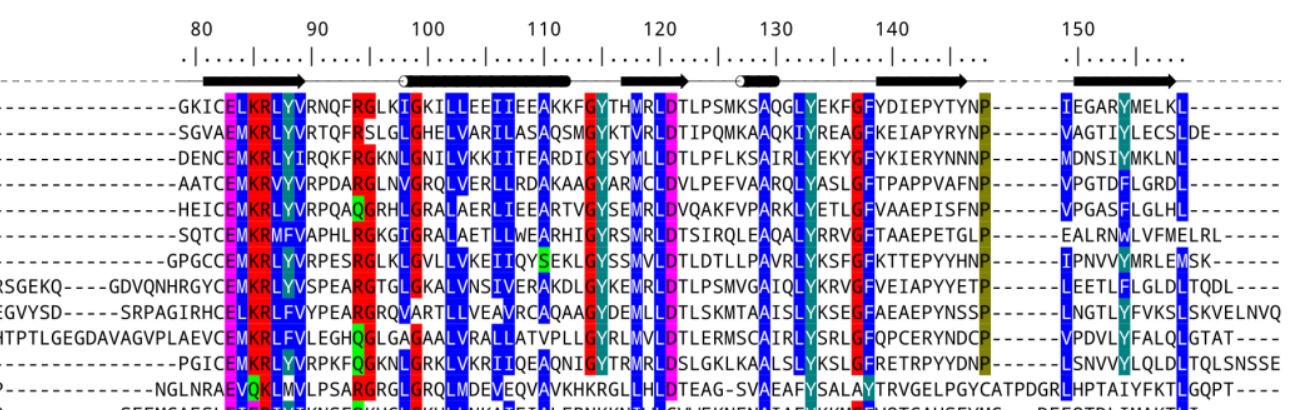




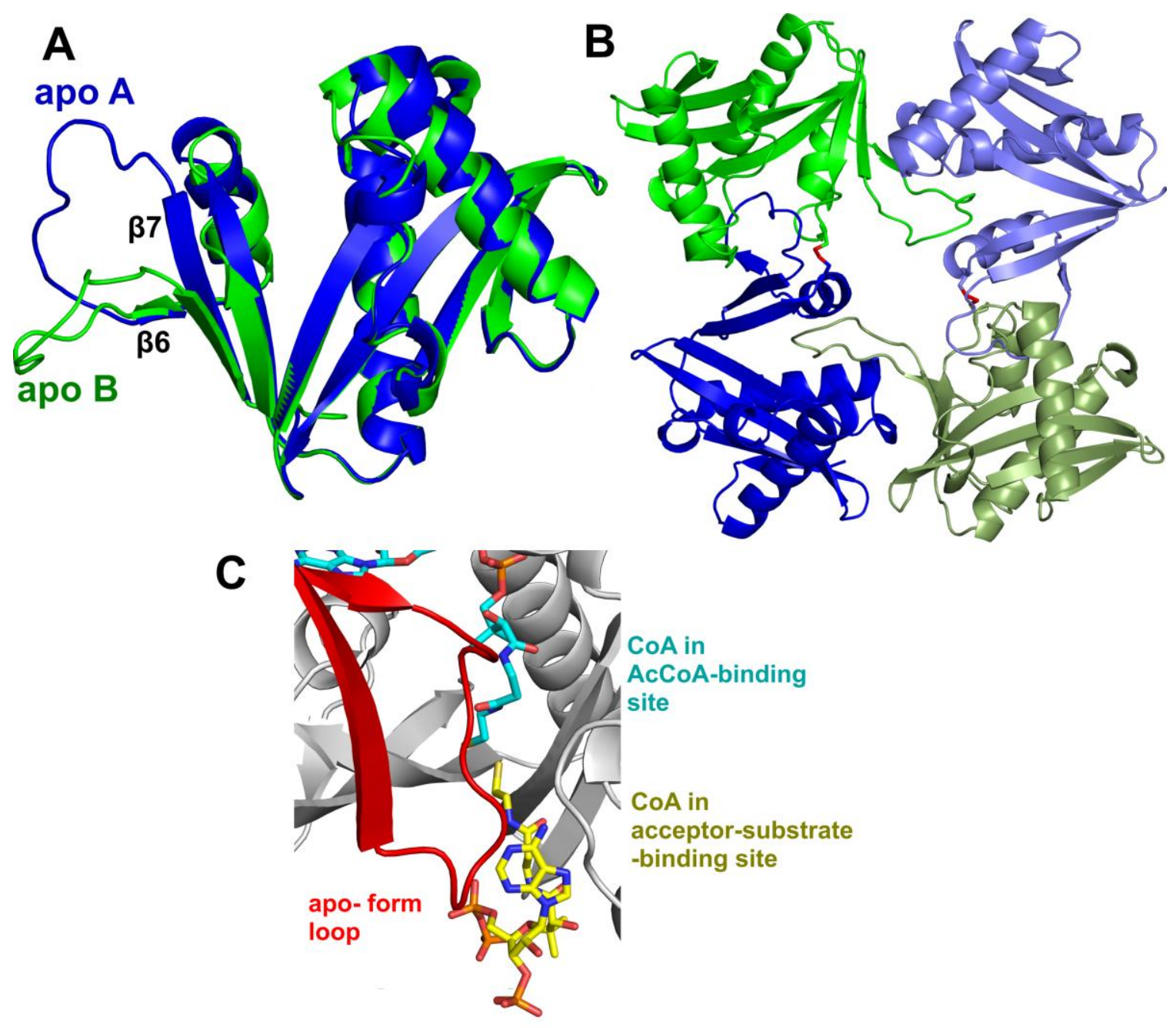



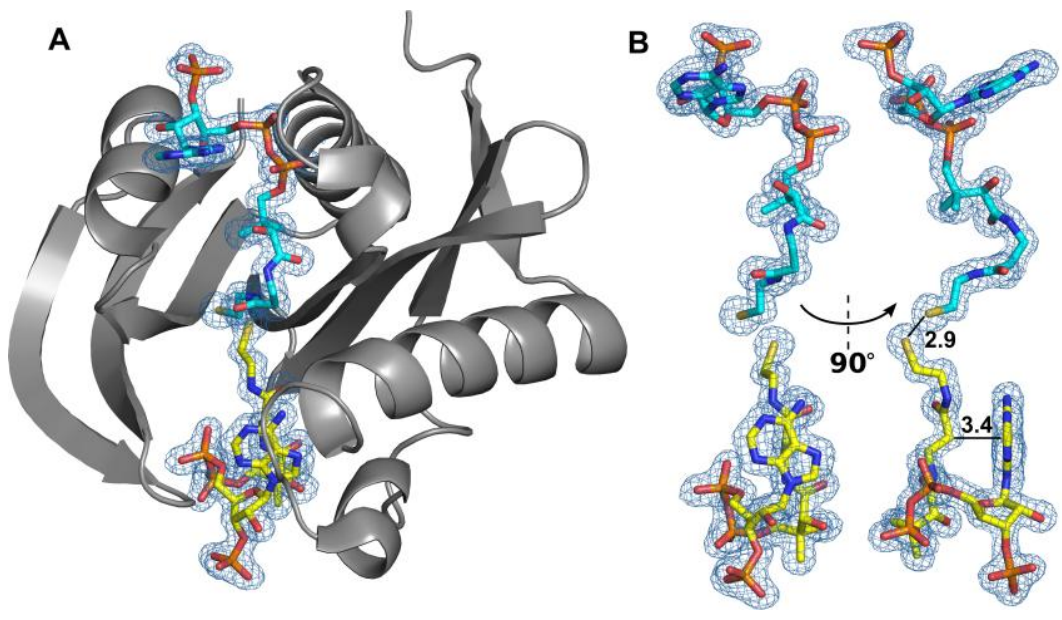

C

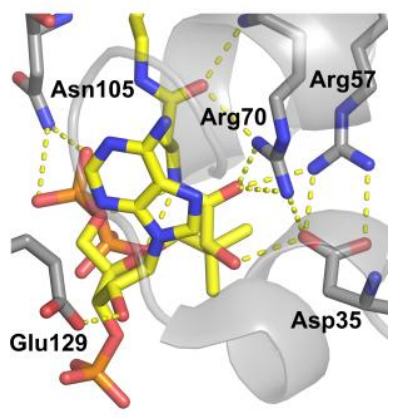



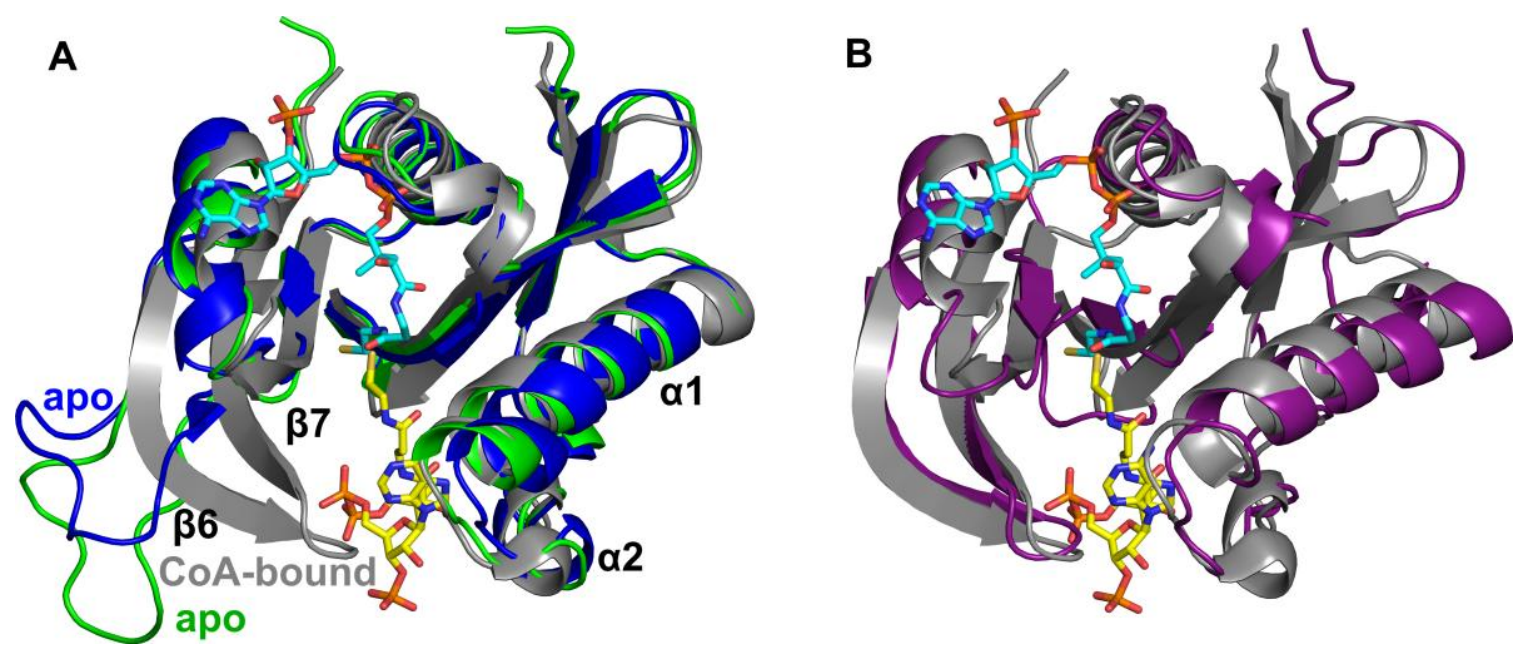


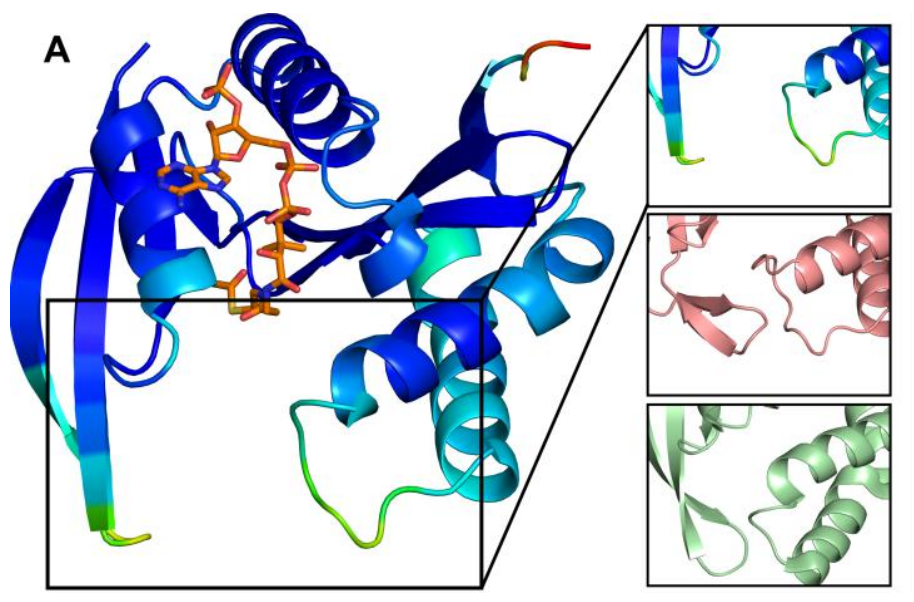

B

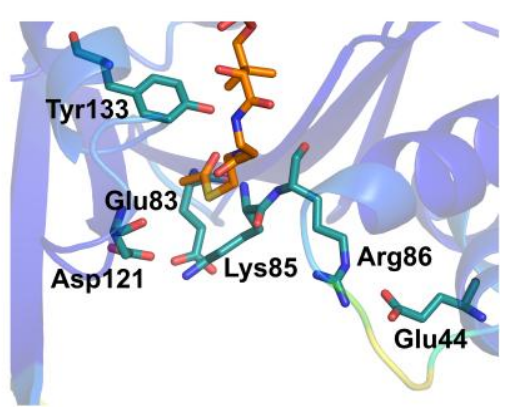




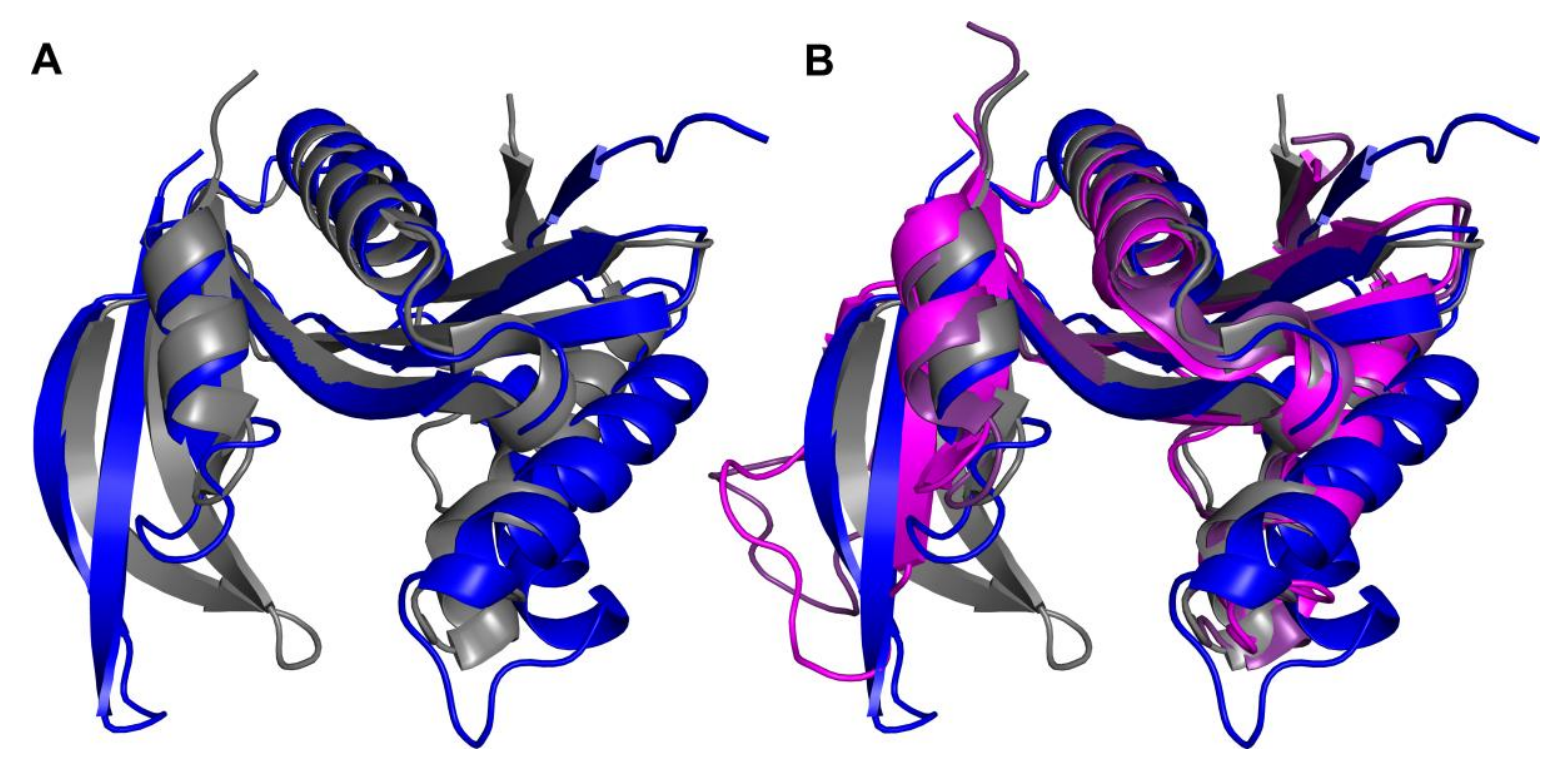



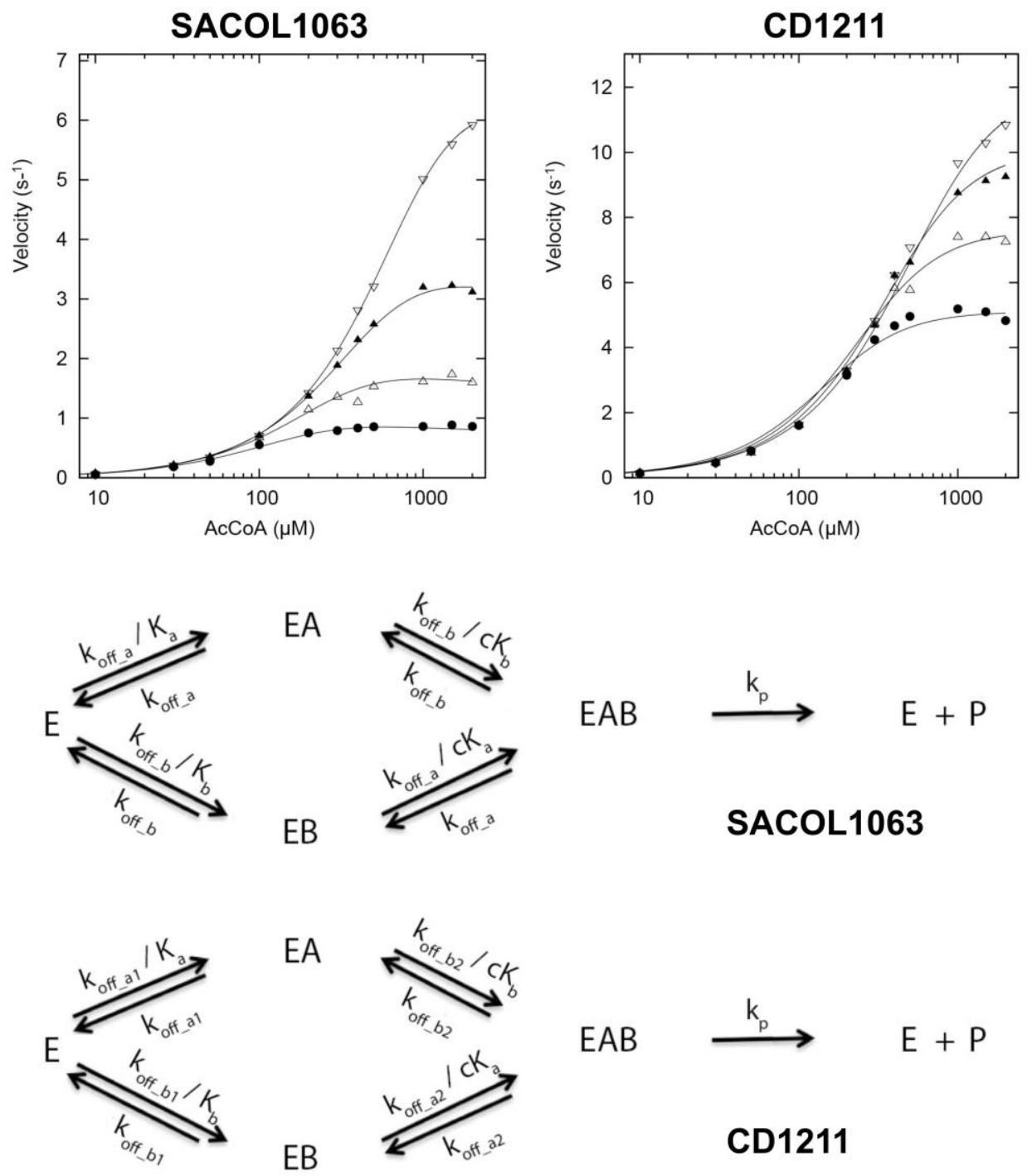


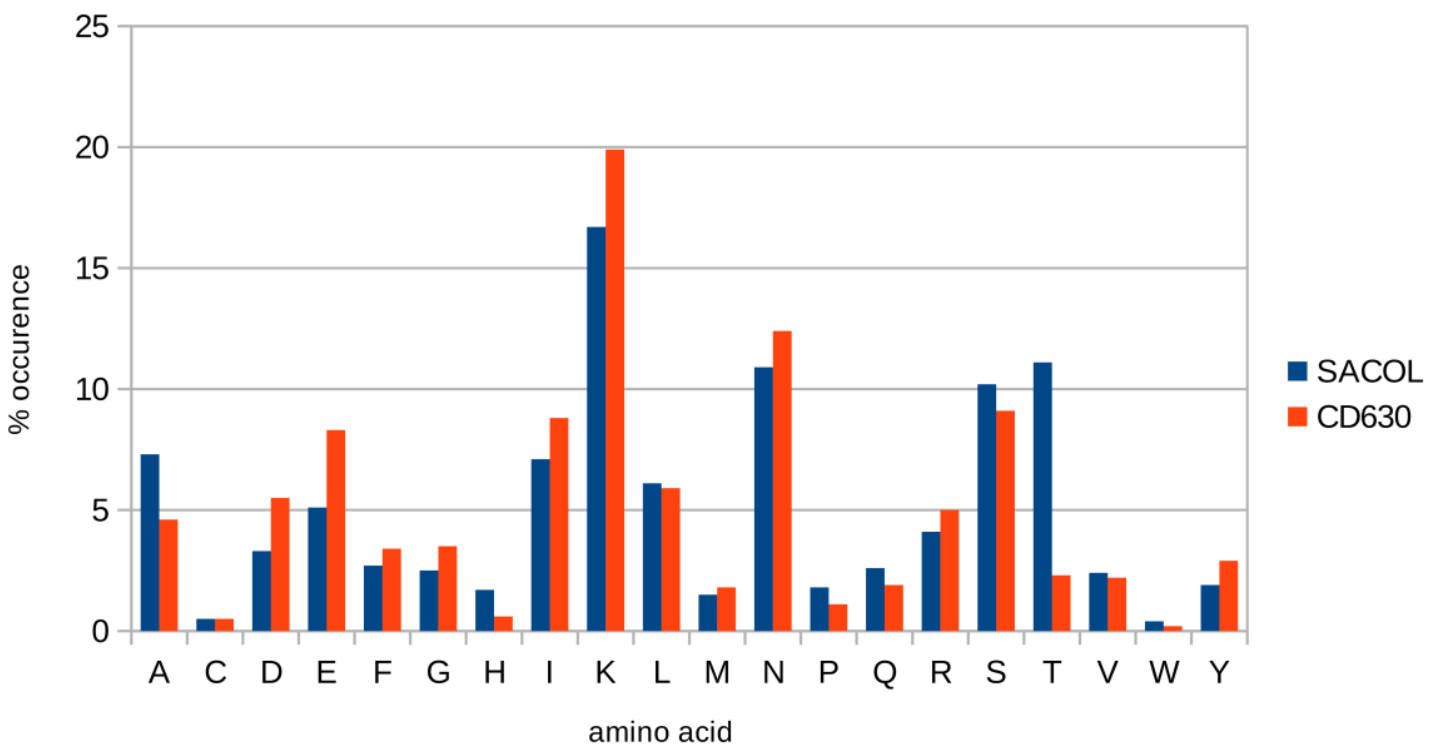


Table 1. Kinetic parameters of SACOL1063 and CD1211 enzymes.

\begin{tabular}{|c|c|c|c|c|c|}
\hline \multicolumn{2}{|c|}{ AcCoA Substrate Saturation Curves } & $\begin{array}{l}\mathrm{k}_{\mathrm{cat}} \\
\left(\mathrm{s}^{-1}\right) \\
\end{array}$ & $\begin{array}{c}\mathrm{S}_{0.5} \\
(\mu \mathrm{M})\end{array}$ & $\mathrm{n}$ & $\begin{array}{l}\text { Catalytic Efficiency } \\
\qquad \begin{array}{c}\mathrm{k}_{\mathrm{cat}} / \mathrm{S}_{0.5} \\
\left(\mathrm{M}^{-1} \mathrm{~s}^{-1}\right)\end{array}\end{array}$ \\
\hline \multicolumn{6}{|l|}{ SACOL1063 } \\
\hline & Constant $[\mathrm{L}-\mathrm{Thr}]^{\mathrm{a}}$ & 5.37 & $419 \pm 22$ & $1.43 \pm 0.08$ & $1.28 \times 10^{4}$ \\
\hline & Constant $[\mathrm{L}-\mathrm{Trp}]^{\mathrm{a}}$ & 0.365 & $72.1 \pm 7.9$ & $1.05 \pm 0.11$ & $5.06 \times 10^{3}$ \\
\hline \multicolumn{6}{|l|}{ CD1211 } \\
\hline & Constant $[\mathrm{L}-\mathrm{Thr}]^{\mathrm{a}}$ & 7.97 & $248 \pm 16$ & $1.56 \pm 0.13$ & $3.09 \times 10^{4}$ \\
\hline & Constant $[\mathrm{L}-\mathrm{Ser}]^{\mathrm{b}}$ & 2.47 & $83.6 \pm 6.8$ & $1.79 \pm 0.20$ & $2.95 \times 10^{4}$ \\
\hline & Constant $[\mathrm{O} \text {-acetyl-L-Ser }]^{\mathrm{b}}$ & 0.447 & $18.9 \pm 1.5$ & $1.91 \pm 0.24$ & $2.37 \times 10^{4}$ \\
\hline & Constant $[\mathrm{L}-\mathrm{Met}]^{\mathrm{b}}$ & 0.245 & $10.8 \pm 0.4$ & $2.90 \pm 0.50$ & $2.27 \times 10^{4}$ \\
\hline \multicolumn{2}{|c|}{ Acceptor Substrate (L-Thr) Saturation Curves } & $\begin{array}{l}\mathbf{k}_{\text {cat }} \\
\left(\mathbf{s}^{-1}\right) \\
\end{array}$ & $\begin{array}{c}\mathbf{S}_{0.5} \\
(\mathbf{m M}) \\
\end{array}$ & $\mathbf{n}$ & $\begin{array}{c}\text { Catalytic Efficiency } \\
\mathbf{k}_{\text {cat }} / \mathbf{S}_{0.5} \\
\left(\mathbf{M}^{-1} \mathbf{s}^{-1}\right) \\
\end{array}$ \\
\hline SACOL1063 & Constant $[\mathrm{AcCoA}]^{\mathrm{c}}$ & 10.3 & $11.6 \pm 0.7$ & $1.14 \pm 0.02$ & $8.90 \times 10^{2}$ \\
\hline CD1211 & Constant $[\mathrm{AcCoA}]^{\mathrm{c}}$ & 8.32 & $5.96 \pm 0.41$ & $1.04 \pm 0.02$ & $1.40 \times 10^{3}$ \\
\hline
\end{tabular}

${ }^{\text {a }}$ Concentration of acceptor substrate was held at $10 \mathrm{mM}$. The data were fitted to a modified Hill equation as described in Materials and Methods over the concentration range of $0-2 \mathrm{mM}$ AcCoA.

${ }^{\mathrm{b}}$ Concentration of acceptor substrate was held at $10 \mathrm{mM}$. The data were fitted to a modified Hill equation as described in Materials and Methods over the concentration range of $0-0.5 \mathrm{mM}$ AcCoA because slight substrate inhibition was seen at higher concentrations of AcCoA.

${ }^{\mathrm{c}}$ Concentration of AcCoA was held at $1 \mathrm{mM}$. The data were fitted to a modified Hill equation as described in Materials and Methods over the concentration range of 0-10 $\mathrm{mM}$ L-Thr. 
Table 2. Crystallographic data and refinement statistics for SACOL1063 structures.

Values in parenthesis refer to the highest resolution shell. AU=asymmetric unit.

PDB code

Data collection

Wavelength $(\AA)$

Unit cell $\left(\AA \stackrel{\circ}{\circ}^{\circ}\right)$

Space group

Resolution range $(\AA)$

Highest resolution shell $(\AA)$

Unique reflections

Redundancy

Completeness $(\%)$

$\mathrm{R}_{\text {merge }}(\%)$

Average $\mathrm{I} / \sigma(\mathrm{I})$

\section{Refinement}

$\mathrm{R}(\%)$

$\mathrm{R}_{\text {free }}(\%)$

Mean B value $\left(\AA^{2}\right)$

B from Wilson plot $\left(\AA^{2}\right)$

RMS deviation bond lengths $(\AA)$

RMS deviation bond angles $\left(^{\circ}\right)$

Ramachandran plot

Most favored regions (\%)
5JQ4 (apo)

5JPH (CoA complex)

$$
\alpha=\gamma=90, \beta=109.5 \quad \alpha=\gamma=90, \beta=97.2
$$

C2

$\mathrm{P} 2_{1}$

$50.0-1.8$

$50.0-1.46$

$1.8-1.83$

$1.46-1.49$

$\mathrm{X}$

$\mathrm{X}$

$4.2(4.1)$

$4.7(4.6)$

$99.9(100.0)$

$99.1(98.3)$

$0.100(0.666)$

$0.060(0.672)$

$26.1(2.1)$

$36.7(2.1)$

18.0

14.2

21.2

17.0

27

21

22.1

20.1

0.02

0.01

1.8

1.5

98.6

98.4 